\title{
Evaluation of Permeability for Columnar Dendritic Structures by Three-dimensional Numerical Flow Analysis
}

\author{
Yukinobu NATSUME, ${ }^{1) *}$ Daiki TAKAHASHI ${ }^{2)}$ Kasumi KAWASHIMA, ${ }^{3)}$ Eiji TANIGAWA ${ }^{3)}$ and Kenichi OHSASA ${ }^{1)}$ \\ 1) Department of Materials Engineering, Akita University, Akita, 010-8502 Japan. \\ 2) Materials Research Laboratory, Kobe Steel Ltd., Kobe, 651-2271 Japan. \\ 3) Business Solution Development Group, Kobelco Systems Co., Kobe, 651-2271 Japan.
}

(Received on September 20, 2013; accepted on November 27, 2013; originally published in Tetsu-to-Hagané, Vol. 99, 2013, No. 2, pp. 117-125)

\begin{abstract}
In order to evaluate the permeability of columnar dendritic structures, three-dimensional (3-D) flow simulations of interdendritic liquid were carried out. The 3-D columnar dendrite morphologies, created by means of a computer-aided design (CAD) software, were based on two-dimensional dendrite morphologies calculated by a phase-field method. The artificial 3-D columnar dendrites were regularly arranged, and six kinds of 3-D columnar dendritic structures were observed, each with different liquid volume fractions between 0.56 and 0.95 . For these 3-D columnar dendritic structures, the flow parallel and normal to the primary arms were calculated using FLUENT, and the permeability of six 3-D columnar dendritic structures for both flow directions were determined using the Darcy law. The values of our simulated permeability were compared with those values of permeability obtained experimentally [K. Murakami, A. Shiraishi and T. Okamoto: Acta Metall. 31 (1983), p. 1417, 32 (1984), p. 1423, C. Y. Liu, K. Murakami and T. Okamoto: Mater. Sci. Tech., 5 (1989), p. 1148]. For both flow directions, our simulated permeability for high liquid volume fractions complemented their experimental permeability for low liquid volume fractions. Therefore, we confirmed the consistency of simulated reading with extrapolations of experimental values of low volume fractions to high volume fractions. In addition, we discussed the limitation of flow within the mushy region, and found that defining the limiting permeability of interdendritic flow, in order to evaluate the relationship between dendritic morphology and the solid volume fraction where interdendritic liquid flow ceases, was effective.
\end{abstract}

KEY WORDS: permeability; columnar dendritic structures; 3-D flow simulation; solidification; macrosegregation.

\section{Introduction}

In large cast iron productions such as ingots and continuous casting slabs, convection within the mushy region plays an important role in the phenomenon of macrosegregation. Macrosegregation represents solute composition inhomogeneity at the macroscopic scale, and can lead to the formation of gross compositional defects that greatly affect the quality of casting products. Therefore, it is important to understand the mechanisms that underlie macrosegregation, such as V-type and A-type segregations, to control it.

V-type segregation is the streak of segregation arranged in a V-shaped pattern at the center of the ingot, and A-type segregation, also called freckle, refers to the streaks oriented almost vertically in an A-shaped pattern at the upper and outer regions of the ingot. Experimental research on the mechanism and conditions that form these segregations have been carried out actively from the 1970s through the 1980s. ${ }^{1-7)}$ Macrosegregation arises mainly from fluid flow and microsegregation during solidification, and it follows

* Corresponding author: E-mail: natsume@gipc.akita-u.ac.jp DOI: http://dx.doi.org/10.2355/isijinternational.54.366 that macrosegregation has to be controlled by controlling solute migration in a molten alloy. Thus, to predict and control macrosegregation, numerical models, which consider solute migration by fluid flow, have been developed since the 1980s. ${ }^{8-13)}$ The flow of interdendritic liquid within the mushy region is important in predicting macrosegregation. The flow of interdendritic liquid can often be described by Darcy's law, and the flow velocity can be defined as follows:

$$
u=-\frac{K}{\varepsilon \mu} \frac{\Delta P}{L}
$$

where $K$ is the permeability, $\varepsilon$ is the volume fraction of interdendritic liquid, $\mu$ is the viscosity, $\Delta P$ is the pressure loss and $L$ is the distance.

The permeability is a very important parameter that affects the flow velocity within the mushy region, and is the parameter that is associated with the region of interdendritic channels. Permeability in equiaxed dendrite structures was first reported by Piwonka and Flemings. ${ }^{14)}$ They measured the permeability of $\mathrm{Al}-4.5 \mathrm{wt} \% \mathrm{Cu}$ alloys by forcing molten lead through interdendritic channels. After that, Apelian et al. ${ }^{15)}$ measured the permeability of $\mathrm{Al}-4 \mathrm{wt} \% \mathrm{Si}$ 
and $\mathrm{Al}-4 \mathrm{wt} \% \mathrm{Si}-0.25 \mathrm{wt} \% \mathrm{Ti}$ alloys by means of having distilled water flow through interdendritic channels. Streat and Weinberg ${ }^{16)}$ measured fluid flow rate though interdendritic channels in $\mathrm{Pb}-20 \mathrm{wt} \% \mathrm{Sn}$ alloys and related the flow to dendrite arm spacing in columnar dendritic structures. In the columnar dendritic structures, the permeability depends on the direction of fluid flow within the mushy region. This means that the values of permeability differ for flow parallel and normal to the primary arms: this is also called the anisotropy of permeability.

Murakami et al. ${ }^{17-19)}$ measured the permeability of a borneol-paraffin organic binary alloy for flow parallel and normal to the primary arms. The permeability of flows parallel, $K_{P}$, and normal, $K_{N}$, to the primary arms were derived from multiple regression analysis as a function of primary dendrite arm spacing (PDAS), $d_{1}$, secondary dendrite arm spacing (SDAS), $d_{2}$, and liquid volume fraction, $g_{L}$.

$$
\begin{gathered}
K_{P}=6.2 \times 10^{-13} d_{1}^{2.2} d_{2}^{-1.7} g_{L}^{3.2} \quad\left(0.19 \leq g_{L} \leq 0.61\right) \ldots . \\
K_{N}=8.8 \times 10^{-19} d_{1}^{1.3} d_{2}^{2.4} g_{L}^{3.2} \quad\left(0.19 \leq g_{L} \leq 0.66\right) \ldots .
\end{gathered}
$$

Liu et $a{ }^{20)}{ }^{20)}$ measured the permeability of borneol-paraffin binary organic alloys for flow parallel and normal to the primary arms with a low liquid volume fraction ranging from 0.19 to 0.22 . The permeability was in fairly good agreement with the value calculated from the empirical equations, Eqs. (2) and (3).

Poirier et $a .^{21-24)}$ also measured the permeability for columnar dendrites of a $\mathrm{Pb}-\mathrm{Sn}$ alloy and derived empirical equations to calculate permeability using multiple regression analysis. These calculations were then verified with the experimental results obtained by them as well as other researchers. In addition, they estimated the permeability for columnar dendritic structures using two-dimensional numerical flow simulations. ${ }^{25,26)}$ Poirier and Heinrich ${ }^{27,28)}$ evaluated the permeability for columnar dendritic structures based on the results from their experiments and simulations. According to them, the permeability for a flow parallel and normal to the primary arms, $K_{P}$ and $K_{N}$, respectively, are given as follows:

$$
K_{P}=\left\{\begin{array}{l}
3.75 \times 10^{-4} g_{L}^{2} d_{1}^{2} \quad\left(g_{L} \leq 0.65\right) \\
2.05 \times 10^{-7}\left[\frac{g_{L}}{1-g_{L}}\right]^{10.739} d_{1}^{2} \quad\left(0.65<g_{L} \leq 0.75\right) \\
0.074\left[\ln \left(1-g_{L}\right)^{-1}-1.49+2\left(1-g_{L}\right)-0.5\left(1-g_{L}\right)^{2}\right] d_{1}^{2} \\
\left(0.75<g_{L} \leq 1.0\right)
\end{array}\right.
$$

$$
K_{N}=\left\{\begin{array}{l}
1.09 \times 10^{-3} g_{L}^{3.32} d_{1}^{2} \quad\left(g_{L} \leq 0.65\right) \\
4.04 \times 10^{-6}\left[\frac{g_{L}}{1-g_{L}}\right]^{6.7336} d_{1}^{2} \quad\left(0.65<g_{L} \leq 0.75\right) \\
{\left[\begin{array}{l}
\left.-6.49 \times 10^{-2}+5.43 \times 10^{-2}\left(\frac{g_{L}}{1-g_{L}}\right)^{0.25}\right] d_{1}^{2} \\
\left(0.75<g_{L} \leq 1.0\right)
\end{array}\right.}
\end{array}\right.
$$

The permeability in these equations is given as a function of $d_{1}$ and $g_{L}$. Poirier and Heinrich formulated the permeability in the case of liquid volume fractions less than 0.65 from experimental data using multiple regression analysis and for liquid volume fractions over 0.75 by numerical flow calculations. For liquid volume fractions between 0.65 and 0.75 , they introduced a relationship in order to best fit the two different equations. This is due to the fact that for liquid volume fractions around 0.7 , the values of permeability obtained from experiment varied greatly from those obtained from simulations. This difference was caused by their use of two-dimensional simulations. Therefore, the relationship between the permeability and the liquid volume fraction must be determined in three dimensions.

Fuloria et al. ${ }^{29)}$ used synchrotron X-ray micro-tomography in order to obtain the three-dimensional columnar dendritic structures of an $\mathrm{Al}-\mathrm{Cu}$ alloy. They also calculated the permeability using flow calculations. There was a reasonable agreement between the calculated and experimentally determined permeability. Madison et al. ${ }^{30)}$ obtained columnar dendritic structures of a Ni-based super alloy by means of scanning the sample using an automated Robo-Met.3D serial sectioning system. They compared the permeability obtained from simulations with the experimental data from various studies, and concluded that their simulated permeability was in general agreement with the permeability reported by various researchers ${ }^{18,19,21,22,29)}$ employing either or both experimental and numerical approaches. However, in the approaches using three-dimensional experimental structures, it still remains difficult to obtain the dendritic structures for higher liquid volume fractions because the permeability will be obtained only for a reduced liquid volume fraction. Therefore, this approach is not sufficient for the evaluation of the permeability for columnar dendritic structures in three dimensions.

In the present work, our purpose is to evaluate quantitatively the permeability for columnar dendritic structures in three dimensions. We created three-dimensional (3-D) columnar dendritic structures from two-dimensional (2-D) dendrite morphology data obtained from phase-field simulations by means of a computer aided design (CAD) software and carried out flow calculations in three dimensions. From the results of the flow calculation, we obtained the permeability of columnar dendrites for relatively higher liquid volume fractions using Darcy's law. To examine the validity of our simulated results, the simulated permeability was compared with the empirical equations to estimate the permeability and the experimental data obtained by Murakami et al. Moreover, we discussed the limitation of flow of the interdendritic liquid for columnar dendritic structures.

\section{Calculation Method}

\subsection{Modeling of Columnar Dendritic Structures in Three Dimensions}

3-D columnar dendritic structures were created from 2-D dendrite morphologies using CAD. To obtain 2-D dendrite morphologies, where the secondary dendrite arms developed, we carried out simulations of the dendrite growth of an Al-Si alloy using the phase-field method. ${ }^{31-33)}$ The details of the calculations were reported in previous work ${ }^{34,35)}$ that investigated the growth of secondary dendrite arms. In the 
present work, the simulated results are of an Al-7 wt $\% \mathrm{Si}$ alloy. 3-D phase-field simulations were not carried out because of high computational costs.

Figure 1 shows an example of 2-D dendrite morphology and single 3-D columnar dendrite created by CAD. Figure 2 shows an example of a 3-D columnar dendritic structure created in the present work. 3-D columnar dendritic structures as shown in Fig. 2 were created as follows: First, a 3$\mathrm{D}$ primary dendrite arm was made by rotating around the $x$ axis forming a cylinder with radius $r$, as shown in Fig. 1(a), where $r$ is the distance between $x$-axis and the broken line. Next, a 3-D secondary dendrite arm was made as a body of rotation having the outline of a 2-D secondary dendrite arm.
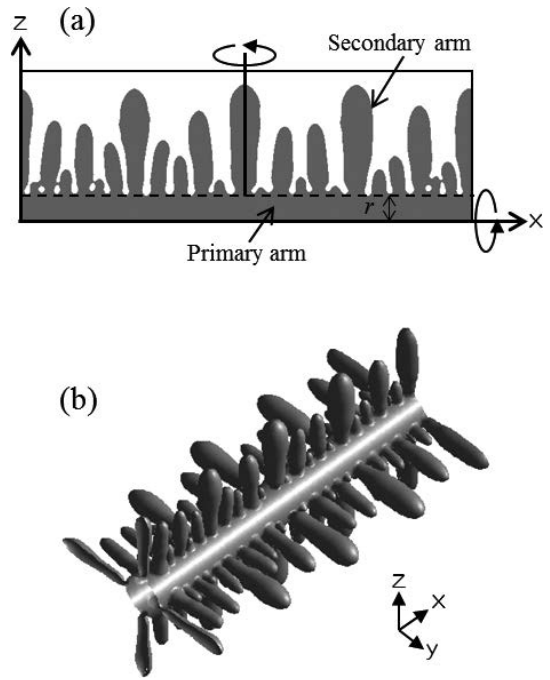

Fig. 1. Dendritic morphology used in the present work. (a) 2-D dendrite calculated by the phase-field method. (b) 3-D dendrite created by CAD (see text for details).

(a)
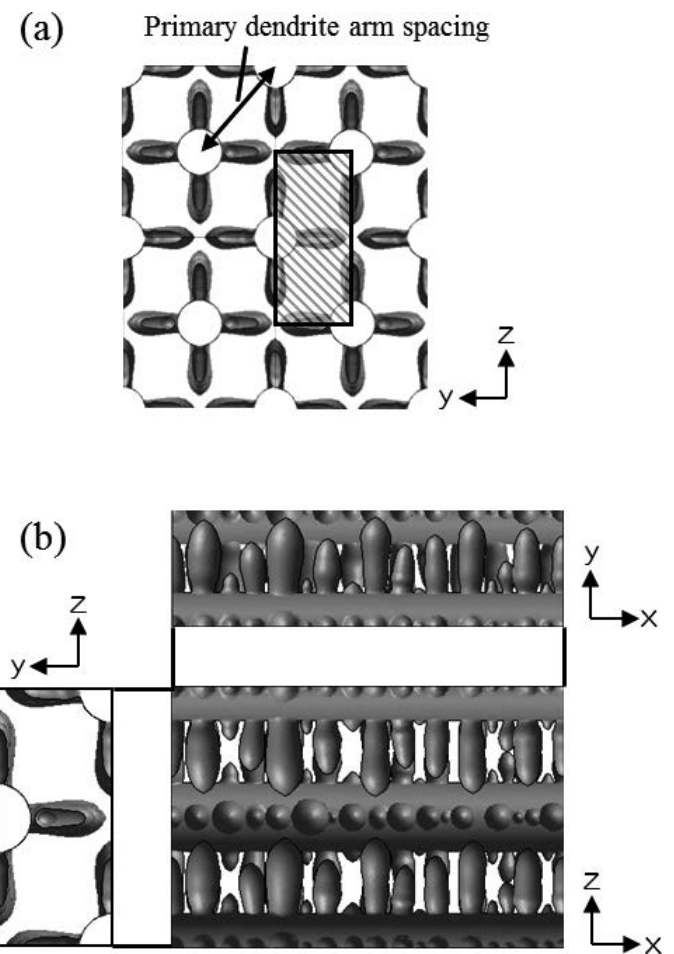

Fig. 2. 3-D columnar dendritic structures used in the present work. (a) Ordered array of columnar dendrites. (b) Post-processing view of the minimum ordered structure.
The axis of rotation was assumed to be the middle axis of the 2-D secondary dendrite arm outline. Rotating around its axis, a 3-D secondary dendrite arm was made and attached to the 3-D primary dendrite arm. By repeating for all of 2D secondary dendrite arms and creating 4-fold symmetry, a single 3-D columnar dendrite was created, as shown in Fig. 1(b). Finally, to create a 3-D columnar dendritic structure, 3-D columnar dendrites were orderly arrayed as shown in Fig. 2(a). The center-to-center distance of primary dendrites corresponds to the PDAS. SDASs for each 3-D columnar dendritic structure were determined by the original 2-D dendrites used to make the 3-D columnar dendrite. A gray hatching area shown in Fig. 2(a) is the minimum ordered structure. Figure 2(b) shows the 3-D minimum ordered structure. The 3-D minimum ordered structures were used as calculation domains for the 3-D flow calculation.

Figure 3 shows the six types of columnar dendritic structures modeled in the present work, with a liquid volume fraction of (a) 0.56 , (b) 0.66 , (c) 0.70 , (d) 0.77 , (e) 0.87 , and (f) 0.95 . The interdendritic region was regarded as the liquid phase. The liquid volume fractions for each 3-D columnar dendritic structure were estimated as the volume ratio of the minimum ordered structure region to the interdendritic region. The liquid volume fractions for columnar dendritic structures were relatively high since the structures were artificially modeled using CAD.
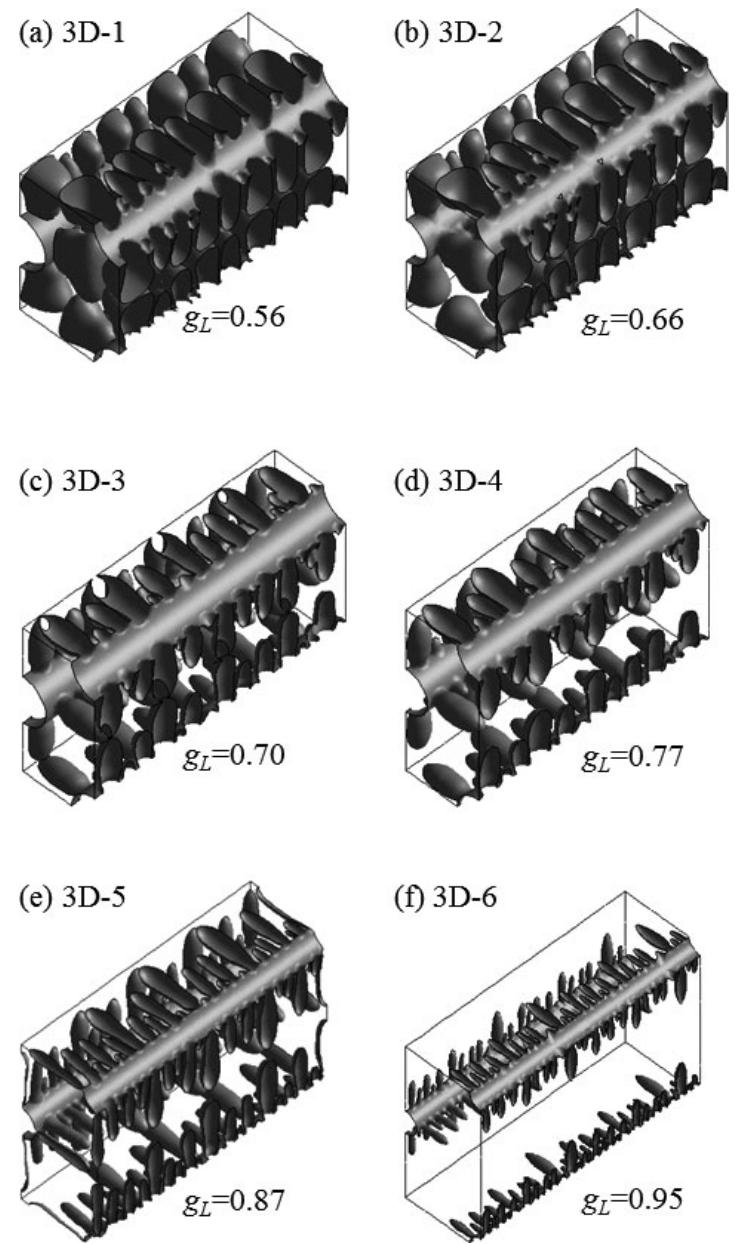

Fig. 3. The three-dimensional columnar dendritic structures modeled using CAD: (a) 3D-1 $g_{L}=0.56$, (b) 3D-2 $g_{L}=0.66$, (c) 3D-3 $g_{L}=0.70$, (d) 3D-4 $g_{L}=0.77$, (e) 3D-5 $g_{L}=0.87$, (f) 3D-6 $g_{L}=0.95$. 


\subsection{Permeability from 3-D Flow Calculations}

As described in Section 1, the permeability for columnar dendritic structures depends on the direction of fluid flow within the mushy region. We carried out the flow calculations in three dimensions using ANSYS FLUENT 6.3. The flow directions were $+\mathrm{x}$ direction for parallel and $-\mathrm{z}$ direction for normal to the primary arms as shown in Fig. 4. In the flow calculations, the superficial velocity, $u_{S}\left(=u_{\text {in }} \times g_{L}\right.$, where $u_{\text {in }}$ is the initial flow velocity), and the pressure loss, $\Delta P$, were calculated and then substituted into Darcy's equation to calculate the permeability for columnar dendritic structures. Darcy's equation is given as follows:

$$
K=\frac{\mu g_{L} u_{S} L}{\Delta P}
$$

where $\mu$ is viscosity and $L=L_{P}$ and $L=L_{N}$, for flows parallel to and normal to the primary arms, respectively. $L_{P}$ and $L_{N}$ are shown in Fig. 4.

Table 1 shows the liquid volume fraction, PDAS, and SDAS for the six types of 3-D columnar dendritic structures shown in Fig. 3. To compare with the experimental data by Murakami et al., ${ }^{18,19)}$ a value of $320 \mu \mathrm{m}$ for the PDAS was used in our simulations. The value of the SDAS for each 3D columnar dendritic structure was determined based on this PDAS $(320 \mu \mathrm{m})$. The values of the SDASs were determined as follows: First, the number of secondary arms, $n_{s}$, is counted from the original 2-D dendrites. Next, $L_{N}$ $\left(=\sqrt{2} d_{1}\right)$ and $L_{P}\left(=a L_{N}\right)$ are determined from 3-D columnar dendritic structures, where $a$ is constant. Therefore, the values of SDASs are determined by $d_{2}=L_{P} / n_{s}=\sqrt{2} a d_{1} / n_{s}$. For these structures, no coarsening and no remelting of the den-

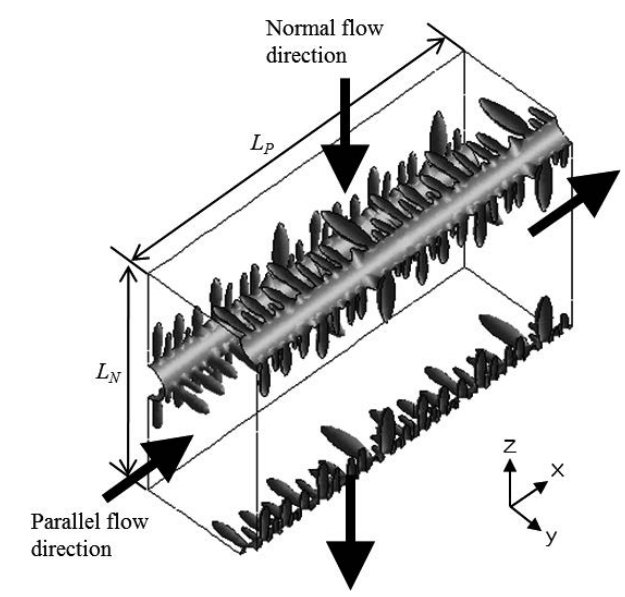

Fig. 4. Parallel and normal flow directions used in the threedimensional flow calculations.

Table 1. Values of the liquid volume fraction and dendrite arm spacing for 3-D columnar dendritic structures.

\begin{tabular}{cccc}
\hline No. & Liquid volume fraction $g_{L}$ & PDAS $d_{1}(\mu \mathrm{m})$ & SDAS $d_{2}(\mu \mathrm{m})$ \\
\hline 3D-1 & 0.56 & 320 & 81.3 \\
3D-2 & 0.66 & 320 & 81.3 \\
3D-3 & 0.70 & 320 & 79.7 \\
3D-4 & 0.77 & 320 & 67.8 \\
3D-5 & 0.87 & 320 & 56.6 \\
3D-6 & 0.95 & 320 & 36.8 \\
\hline
\end{tabular}

drite arms were assumed.

The boundary conditions for the flow calculations are as follows: In parallel flow, the y-z plane had a periodic condition, and the $\mathrm{x}-\mathrm{y}$ and $\mathrm{x}-\mathrm{z}$ planes had a symmetry condition. In normal flow, the $x-y$ plane had a periodic condition, and the $y-z$ and $x-z$ planes had a symmetry condition. Solidliquid interface for dendritic structures was set on no slip wall.

The regions of interdendritic liquid for each columnar dendritic structure were meshed into unstructured tetragonal divisions using ANSYS ICEM CFD. The number of partitions in each columnar dendritic structure was in the range of 0.4-0.8 million. In the flow calculations, the interdendritic liquid was assumed to be incompressible. An initial flow velocity of $1 \times 10^{-4} \mathrm{~m} / \mathrm{s}$ was used for all calculation conditions. To carry out the flow calculations, the viscosity, $\mu$, and the density, $\rho$, were required as material properties. In the present work, the values of $\mu$ and $\rho$ of pure iron ${ }^{36)}$ were used: $\mu=5.5 \times 10^{-3} \mathrm{~Pa} \mathrm{~s}$ and $\rho=7.015 \times 10^{3} \mathrm{~kg} / \mathrm{m}^{3}$. Thus, the kinematic viscosity of iron, $v$, is $7.84 \times 10^{-7} \mathrm{~m}^{2} / \mathrm{s}$. To confirm that the material properties do not affect the permeability estimated from the simulation results, we carried out the simulations for a different kinematic viscosity $(2.64 \times$ $\left.10^{-7} \mathrm{~m}^{2} / \mathrm{s}\right)$ which was estimated from the viscosity $(1.85 \times$ $\left.10^{-3} \mathrm{~Pa} \mathrm{~s}\right)$ and the density $\left(7.000 \times 10^{3} \mathrm{~kg} / \mathrm{m}^{3}\right)$ of pure tin. The kinematic viscosity of pure iron was about three times larger than that of pure tin.

\section{Results and Discussion}

\subsection{Simulated Permeability for Columnar Dendritic Structures}

Figure 5 shows the contour planes of flow velocity for $x-$ $z$ cross sections of the columnar dendritic structure of 3D4. For flow parallel to the primary arms, the flow velocities between the primary arms were considerably high, and the ones between the secondary arms were very low. Conversely, for the flow normal to the primary arms, flows were observed between the secondary arms with wide spacing. However, the maximum flow velocity for the normal direction was considerably lower than that of parallel direction. Therefore, it was noted that the permeability for the flow parallel to the primary arms depends on the PDAS, and that for the flow normal to the primary arms depends on both the PDAS and SDAS

Table 2 shows the values of the simulated permeability for flow parallel and normal to the primary arms for each columnar dendritic structure. The simulated permeability increased with increasing liquid volume fraction for both flow directions. The simulated permeability for flow parallel to the primary arms was several times as larger than for flow normal to the primary arms. From these results, we confirmed that there was an anisotropy of permeability for the columnar dendritic structures.

Table 3 shows the values of the simulated permeability estimated by two kinematic viscosities for columnar dendritic structures of 3D-1 $\left(g_{L}=0.56\right)$ and 3D-6 $\left(g_{L}=0.95\right)$. Since this calculation was done to examine the influence, if any, of material properties on the permeability, the structure having the highest and the lowest liquid volume fraction were selected. For the liquid volume fractions of both 0.56 

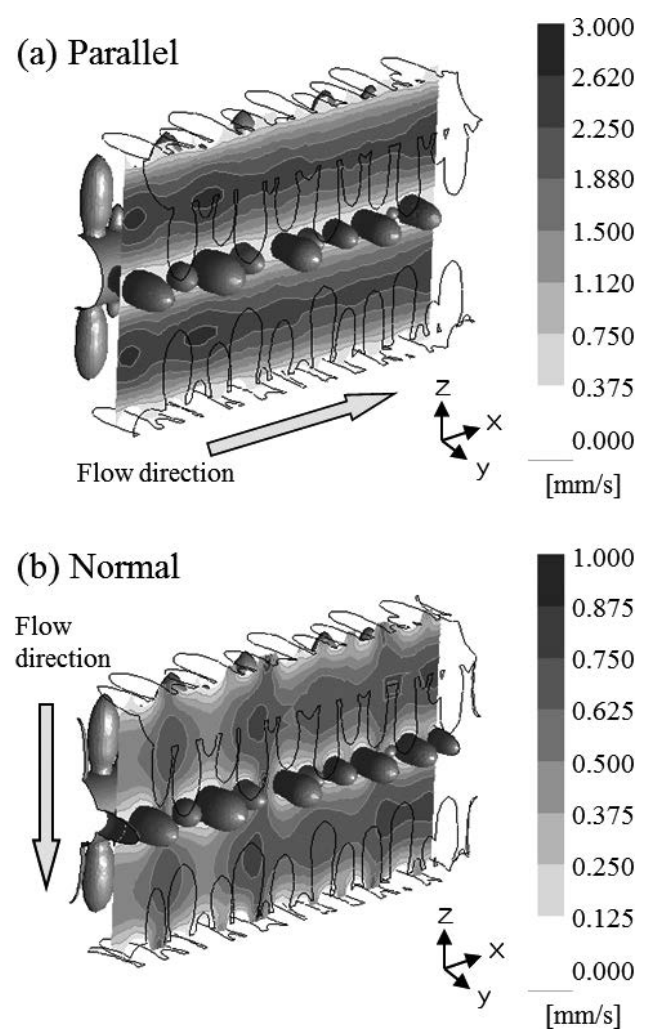

Fig. 5. Contour plots of flow velocity for $x-z$ cross sections of the 3D-4 structure. (a) For flow parallel to the primary dendrites. (b) For flow normal to the primary dendrites.

Table 2. Values of our simulated permeability for flows parallel and normal to the primary arms.

\begin{tabular}{cccc}
\hline \multirow{2}{*}{ No. } & Liquid volume fraction $g_{L}$ & \multicolumn{2}{c}{ Permeability } \\
\cline { 3 - 4 } & & $K_{P}\left(\mathrm{~m}^{2}\right)$ & $K_{N}\left(\mathrm{~m}^{2}\right)$ \\
\hline 3D-1 & 0.56 & $1.08 \times 10^{-10}$ & $5.68 \times 10^{-11}$ \\
3D-2 & 0.66 & $3.70 \times 10^{-10}$ & $9.34 \times 10^{-11}$ \\
3D-3 & 0.70 & $6.13 \times 10^{-10}$ & $1.32 \times 10^{-10}$ \\
3D-4 & 0.77 & $7.97 \times 10^{-10}$ & $2.82 \times 10^{-10}$ \\
3D-5 & 0.87 & $1.13 \times 10^{-9}$ & $3.77 \times 10^{-10}$ \\
3D-6 & 0.95 & $4.30 \times 10^{-9}$ & $2.29 \times 10^{-9}$ \\
\hline
\end{tabular}

Table 3. Values of our simulated permeability calculated by two different kinematic viscosities.

\begin{tabular}{ccccc}
\hline \multirow{2}{*}{$\begin{array}{c}\text { No. } \\
\text { (Element) }\end{array}$} & \multirow{2}{*}{$\begin{array}{c}\text { Liquid volume } \\
\text { fraction } g_{L}\end{array}$} & $\begin{array}{c}\text { Kinematic } \\
\text { viscosity }\left(\mathrm{m}^{2} / \mathrm{s}\right)\end{array}$ & \multicolumn{2}{c}{ Permeability } \\
\cline { 4 - 5 } & & $K_{P}\left(\mathrm{~m}^{2}\right)$ & $K_{N}\left(\mathrm{~m}^{2}\right)$ \\
\hline 3D-1 (Fe) & 0.56 & $7.84 \times 10^{-7}$ & $1.08 \times 10^{-10}$ & $5.68 \times 10^{-11}$ \\
3D-1 (Sn) & 0.56 & $2.64 \times 10^{-7}$ & $1.08 \times 10^{-10}$ & $5.67 \times 10^{-11}$ \\
& & & & \\
3D-6 (Fe) & 0.95 & $7.84 \times 10^{-7}$ & $4.30 \times 10^{-9}$ & $2.29 \times 10^{-9}$ \\
3D-6 (Sn) & 0.95 & $2.64 \times 10^{-7}$ & $4.29 \times 10^{-9}$ & $2.27 \times 10^{-9}$ \\
\hline
\end{tabular}

and 0.95 , the values of simulated permeability for the kinematic viscosity of iron were in fairly good agreement with those of tin. From these results, we confirmed that our flow simulations to determine permeability for columnar dendritic structures were valid.

\subsection{Comparison with the Permeability by Other Equa- tions}

In this section, we compared our simulated permeability with the permeability that calculated by two empirical equations and one physical model. Two empirical equations are Eqs. (2) and (3) by Murakami et al. (Murakami's equation) and Eqs. (4) and (5) by Poirier and Heinrich (PoirierHeinrich's equation). The physical model is a model based on the Hargen-Poiseuille law (Hargen-Poiseuille model).

The permeability obtained from the Hargen-Poiseuille model is often used to compare with the experimentally determined value. ${ }^{14,16,22)}$ In the Hargen-Poiseuille law, the flow velocity, $V$, as it flows through a tube with radius $R$ is described as follows:

$$
V=-\frac{R^{2}}{8 \mu} \frac{d P}{d x}
$$

For the flow of interdendritic liquid parallel and normal to the primary arms, we assumed the simplified columnar dendritic structure as shown in Fig. 6. The cross-sectional area of one channel for flow parallel to the primary arms, $A_{P}$, and the one for flow normal to the primary arms, $A_{N}$, were as follows:

$$
\begin{aligned}
& A_{P}=\left(\frac{d_{1}}{\sqrt{2}}\right)^{2} g_{L} \\
& A_{N}=\left(\frac{d_{1} d_{2}}{\sqrt{2}}\right) g_{L}
\end{aligned}
$$

$A_{P}$ was described by the PDAS and liquid volume fraction, and $A_{N}$ was described by the PDAS, SDAS, and liquid volume fraction. Assuming that the cross-sectional area of one tube, $\pi R^{2}$, is equal to $A_{P}$ or $A_{N}$, the flow velocity for the flow parallel to the primary arms, $V_{P}$, and normal to the primary arms, $V_{N}$, were as follows:

$$
\begin{aligned}
& V_{P}=-\frac{g_{L}}{8 \pi \mu}\left(\frac{d_{1}}{\sqrt{2}}\right)^{2} \frac{d P}{d x} . \\
& V_{N}=-\frac{g_{L}}{8 \pi \mu}\left(\frac{d_{1} d_{2}}{\sqrt{2}}\right) \frac{d P}{d y} .
\end{aligned}
$$

Using Darcy's equation, the permeability for flow parallel and normal to the primary arms was described as follows:

$$
\begin{gathered}
K_{P}=\frac{g_{L}^{2} d_{1}^{2}}{16 \pi} \\
K_{N}=\frac{g_{L}^{2} d_{1} d_{2}}{8 \pi \sqrt{2}}
\end{gathered}
$$

These equations are the Hargen-Poiseuille model used in the present work.

Figure 7 shows the relationship between permeability and liquid volume fraction calculated by our simulations, Murakami's equation, Poirier-Heinrich's equation, and the Hargen-Poiseuille model. First, a comparison of the permeability that obtained by our simulations and that calculated by Murakami's equation was done. For parallel flow, the permeability gradually increased with increasing liquid volume fraction. However, the permeability in our simulations was about ten times greater than that of Murakami's equation. On the other hand, for normal flow, the permeability from Murakami's equation decreased with increasing liquid volume fraction when the liquid volume fraction was exceeded 0.7. This tendency is not accurate since the per- 
(a) Parallel

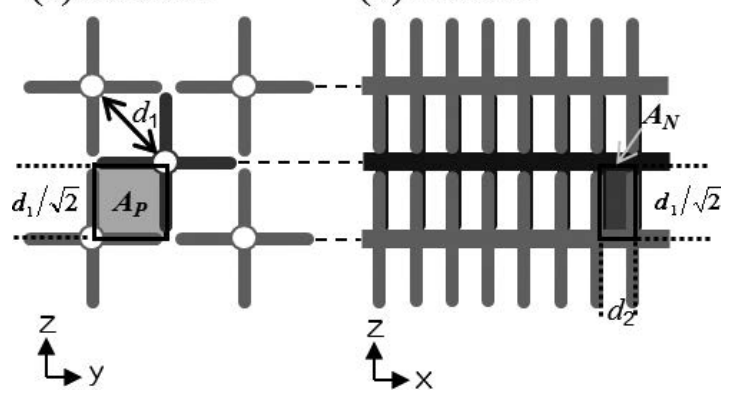

Fig. 6. Schematic illustrations of columnar dendritic structures assumed to derive the Hargen-Poiseuille's equation used to evaluate our simulated permeabilities from HargenPoiseuille's law. (a) For flow parallel to the primary dendrites. (b) For flow normal to the primary dendrites.
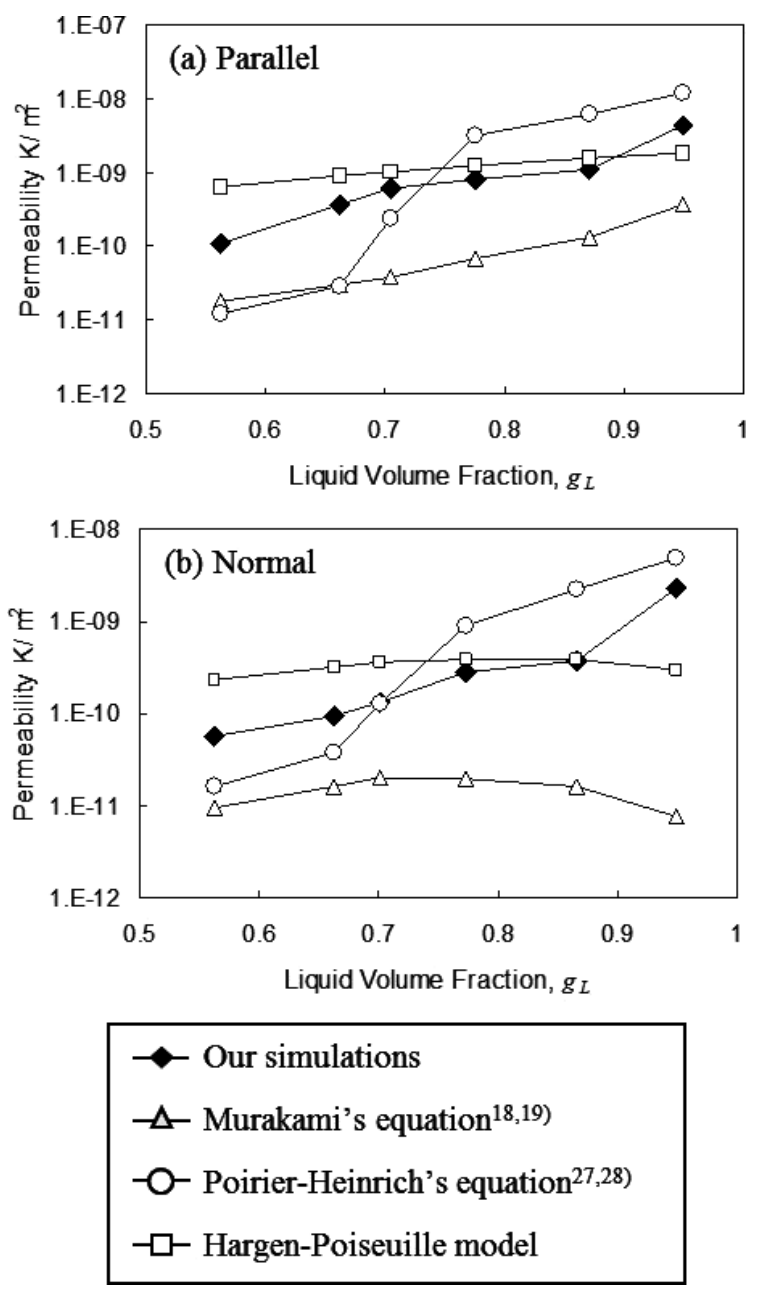

Fig. 7. Relationship between the permeability and the liquid volume fraction for our simulations and other equations. (a) Flow parallel to the primary arms. (b) Flow normal to the primary arms.

meability must increase with increasing liquid volume fraction and the values have to tend to be infinity for $g_{L}=1$. The reason why this decrease was obtained is that the permeability obtained from Murakami's equation was extrapolated for liquid volume fractions over 0.66 . Thus, for higher liquid volume fractions, it is difficult to determine accurately the permeability using Murakami's equation.

Next, a comparison of the permeability obtained by our simulations and that calculated by Poirier-Heinrich's equation was done. In both parallel and normal flow, the permeability gradually increased with increasing liquid volume fraction. The permeability by Poirier-Heinrich's equation varied artificially for liquid volume fractions between 0.65 and 0.75 . Thus, for a liquid volume fraction of less than 0.65 , the values of permeability from our simulations were larger than the values of permeability obtained by PoirierHeinrich's equation, and for a liquid volume fraction over 0.75 , the values from our simulations were smaller than those obtained by Poirier-Heinrich's equation. The reason why the permeability obtained by Poirier-Heinrich's equation varies artificially is that simulated results in two dimensions are used to determine the permeability for high liquid volume fractions. The liquid volume fraction measured from 3 -D dendritic structures is not equal to that measured from 2-D dendritic structures, and it is very difficult to accurately estimate the liquid volume fraction in the three dimensions from that in two dimensions. The relationship between the permeability and liquid volume fraction estimated in their 2D simulations might be correct. Therefore, Poirier-Heinrich's equation is not sufficient in determining the permeability for higher liquid volume fractions.

Finally, a comparison of the permeability obtained by our simulations and that calculated by the Hargen-Poiseuille model was done. In parallel flow, the values of permeability obtained by our simulations were in good agreement with those obtained by the Hargen-Poiseuille model. From this result, it is noted that the flow of interdendritic liquid for higher liquid volume fraction was similar to the flow through a tube. On the other hand, in normal flow, similar to Murakami's equation, the permeability obtained by the Hargen-Poiseuille model slightly decreased with increasing liquid volume fraction for liquid volume fractions over 0.7 . Moreover, in both parallel and normal flow, the values of permeability obtained by the Hargen-Poiseuille model remained large for relatively low liquid volume fractions. From these results, it is noted that the Hargen-Poiseuille model cannot sufficiently determine the permeability for flow through complicated channels, such as for the normal direction, or flow for a low liquid volume fraction.

By comparing our simulated permeability with two empirical equations and one physical model, we confirmed that our simulated permeability was generally valid. To investigate the validity of our simulations in detail, we compared them with experimental data obtained by Murakami et al. ${ }^{18-20)}$ (Murakami's group) in the next section.

\subsection{Comparison with the Experimental Data by Murakami's Group}

In this section, in order to confirm whether the permeability obtained by our simulations was quantitatively valid or not, we compared them with the experimental data obtained by Murakami's group. ${ }^{18-20)}$

Murakami's group measured the permeability of borneolparaffin organic binary alloys for flows parallel to and normal to the primary arms. In their experiments, the permeability of columnar dendritic structures for liquid volume fractions between 0.19 and 0.66 was investigated. Compared with other studies ${ }^{16,21,22)}$ that experimentally measured the permeability, their investigation included a wider range of volume 
fractions. Hence, sufficient experimental data is available to validate our simulated permeability.

Figure 8 shows the relationship between the permeability and the liquid volume fraction for our simulations and the experiments by Murakami's group. In our simulations, the values of liquid volume fraction ranged from 0.56 to 0.95 for flow parallel and normal to the primary arms. On the other hand, in the experiments, the values of liquid volume fraction ranged from 0.19 to 0.53 for flow parallel to the primary arms and ranged from 0.19 to 0.62 for flow normal to the primary arms. The plots of the permeability from our simulations complemented that of the experiments missing for high liquid volume fractions. We obtained the following correlations between permeability and liquid volume fraction:

$$
\begin{aligned}
& K_{P}=5.50 \times 10^{-14} \exp \left(12.0 g_{L}\right) \\
& K_{N}=5.68 \times 10^{-14} \exp \left(10.7 g_{L}\right)
\end{aligned}
$$

These correlations showed that the simulated permeability was quantitatively valid for high liquid volume fraction. In addition, since these equations were obtained for columnar dendritic structures with a PDAS of $320 \mu \mathrm{m}$, there should be a similar relationship for different PDAS values.

\subsection{Discussion for the Limitation of Flow within the Mushy Region}

Compared with the full liquid region, flow of interden-
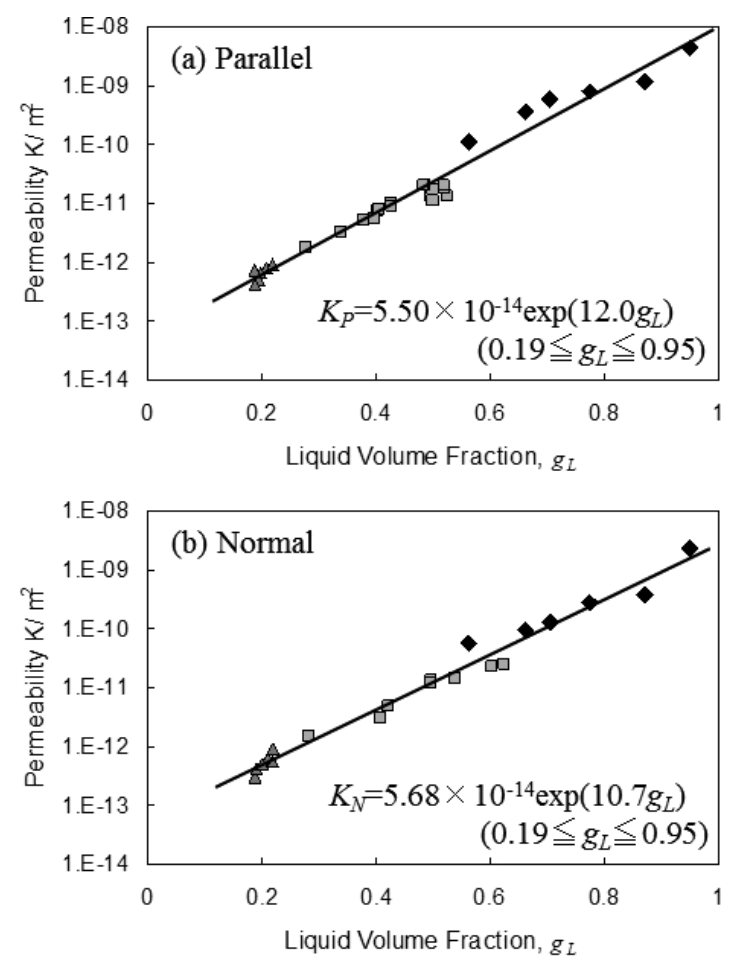
- Our simulations
$\square$ Experiment: Murakami et al. ${ }^{18,19)}$
$\Delta$ Experiment: Liu et al. ${ }^{20}$ )

Fig. 8. Relationship between the permeability and liquid volume fraction for our simulations and the experiments by Murakami et al. ${ }^{18,19)}$ and Liu et al. ${ }^{20)}$ dritic liquid within the mushy region becomes difficult. When the interdendritic liquid was fully surrounded by a solid phase during solidification, the interdendritic liquid could no longer flow within the mushy region. At this time, the solid volume fraction is not one, i.e. there is not yet a fully solid state. We refer to the solid volume fraction for such a situation as "the limiting solid fraction." The limiting solid fraction has been reported in many studies. Takahashi et al. ${ }^{37,38)}$ investigated the relationship between the fluidity and the limitation of flow in the mushy region in $\mathrm{Al}-\mathrm{Cu}$ and $\mathrm{Al}-\mathrm{Si}$ alloys, and theoretically estimated that the value of the limiting solid fraction was 0.67 . In experiments on carbon steel by Takahashi et al., ${ }^{39)}$ it was reported that the values of limiting solid fraction were in the range of 0.60 to 0.73 because of dendritic structures formed under different cooling rates. This means that the limiting solid fraction relates to the dendritic microstructures forming within the mushy region. However, it is difficult to determine the specific values of the limiting solid fraction for a specific microstructure. Therefore, we suggest using the permeability when the interdendritic liquid becomes impossible to flow within the mushy region instead of the limiting solid fraction (called "the limiting permeability" in this discussion), and discussed the validity of this definition.

Figure 9 shows the relationship between the permeability and the solid volume fraction obtained by Eqs. (14) and (15) for a PDAS of $320 \mu \mathrm{m}$. Assuming that the limiting permeability was $1 \times 10^{-10} \mathrm{~m}^{2}$, the values of the limiting solid fraction were in the range of 0.301 to 0.374 ; the limiting solid fractions for flow parallel and normal to the primary arms were 0.374 and 0.301 , respectively. Moreover, assuming that the limiting permeability was $1 \times 10^{-11} \mathrm{~m}^{2}$, the values of the limiting solid fraction were in the range of 0.516 to 0.566; the limiting solid fractions for flow parallel and normal to the primary arms were 0.566 and 0.516 , respectively. From the results, it was noted that the limiting solid fraction varied considerably with the permeability. As mentioned in section 3.3, the correlations such as Eqs. (14) and (15), can be obtained for various PDASs. Thus, the relationship between the limiting solid fraction and the PDAS can be obtained for various PDASs.

Figure 10 shows the relationship between the solid volume fraction and the PDAS when values of the limiting permeability of $1 \times 10^{-10} \mathrm{~m}^{2}$ and $1 \times 10^{-11} \mathrm{~m}^{2}$ were assumed. From these results, if the limiting permeability was $1 \times 10^{-10} \mathrm{~m}^{2}$,

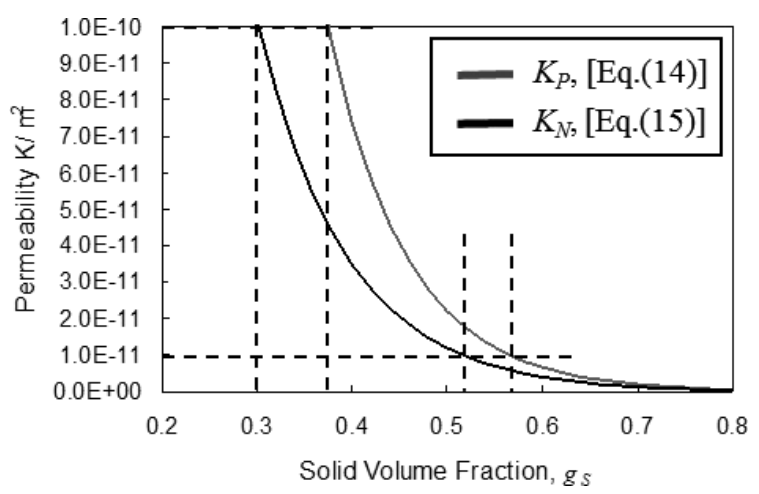

Fig. 9. Relationship between the permeability and solid volume fraction calculated by Eqs. (14) and (15). The range of permeability from $0 \mathrm{~m}^{2}$ to $1.0 \times 10^{-10} \mathrm{~m}^{2}$ is shown. 

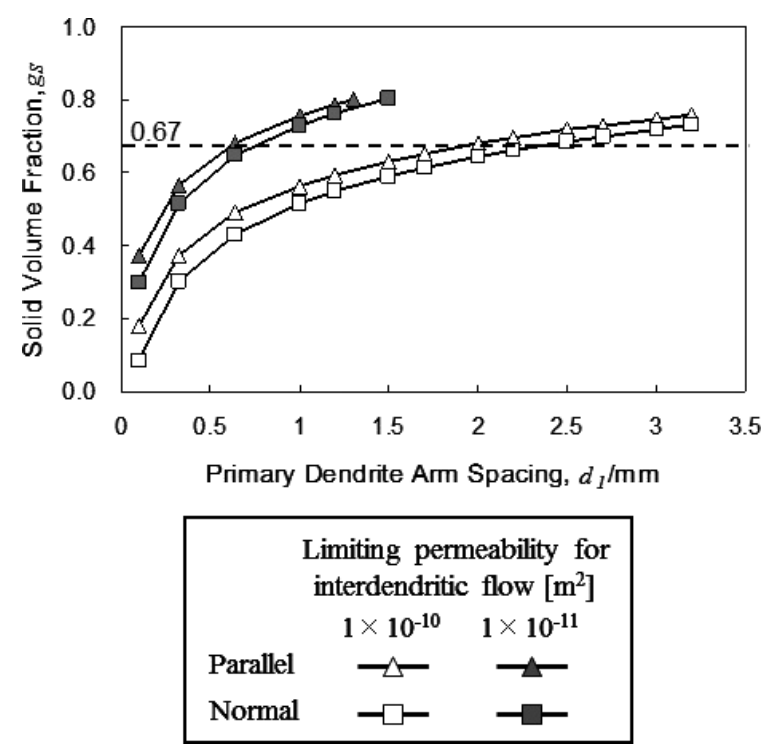

Fig. 10. Relationship between the primary dendrite arm spacing and the solid volume fraction for the limiting permeability when the values of $1 \times 10^{-10} \mathrm{~m}^{2}$ and $1 \times 10^{-11} \mathrm{~m}^{2}$ are assumed.

the values of the PDAS for the limiting solid fraction of 0.67 were in the range of 2 to $2.5 \mathrm{~mm}$. Assuming that the value of the SDAS is about 1/4 of that of the PDAS, the values of the SDAS estimated from these results were in good agreement with those measured by the experiments of Takahashi et $a l .^{37,38)}$ In fact, we cannot determine whether the value of the limiting permeability is $1 \times 10^{-10} \mathrm{~m}^{2}$ or not. However, the discussion in this section helps deepen our understanding about the limitation of flow for interdendritic structures. Moreover, in order to quantitatively predict macrosegregation using these simulations, we must investigate in detail the relationship between the limiting solid fraction (Or the limiting permeability) and the dendritic structures.

\section{Conclusions}

In order to evaluate quantitatively the permeability of columnar dendritic structures, we carried out flow calculations for 3-D columnar dendritic structures that were created from 2-D dendrite morphologies calculated by the phasefield method using CAD. From the results of the calculations for flow, parallel and normal to the primary arms, the values of the permeability of 3-D columnar dendritic structures were calculated by Darcy's law. The permeability that was obtained by our simulations compared with that obtained by Murakami's equations, Poirier-Heinrich's equations and the Hargen-Poiseuille model. We confirmed that our simulated permeability was in general agreement with their permeability. In addition, to confirm that our simulations were quantitatively valid, we compared them with experimental data obtained by Murakami's group. ${ }^{18-20)}$ The permeability obtained by the simulations complemented that obtained by their experiments, which lacked data at high liquid volume fraction. We obtained a good correlation between the simulated and experimental permeability using an exponential approximation. Finally, we discussed the limitation of flow within the mushy region. By defining the permeability at which the flow of interdendritic liquid become impossible (the limiting permeability), we confirmed that there is a relationship between dendritic morphology and the solid volume fraction, as evidenced by the limiting solid fraction, which is the solid fraction at which the flow of interdendritic liquid become impossible.

\section{REFERENCES}

1) K. Tashiro, T. Tdoroki and S. Kimura: Tetsu-to-Hagané, 57 (1971), 1479 .

2) K. Suzuki and T. Miyamoto: Tetsu-to-Hagané, 63 (1977), 53.

3) K. Suzuki and T. Miyamoto: Tetsu-to-Hagané, 65 (1979), 1571.

4) N. Mori, N. Sasaguri, K. Ogi and K. Matsuda: J. Jpn. Inst. Met., 50 (1986), 480.

5) N. Mori and K. Ogi: J. Jpn. Inst. Met., 50 (1986), 486.

6) H. Yamada, T. Sakurai and T. Takenouchi: Tetsu-to-Hagané, 75 (1989), 97.

7) H. Yamada, T. Sakurai and T. Takenouchi: Tetsu-to-Hagané, 75 (1989), 105.

8) I. Ohnaka and K. Kobayashi: Trans. Iron Steel Inst. Jpn., 26 (1986), 781.

9) P. Nandapurkar, D. R. Poirier, J. C. Heinrich and S. Felicelli: Metall. Trans. B, 20B (1989), 711.

10) S. D. Felicelli, J. C. Heinrich and D. R. Poirier: Metall. Trans. B, 22B (1991), 847.

11) C. Beckermann, J. P. Gu and W. J. Boettinger: Metall. Mater. Trans. $A$, 31A (2000), 2545.

12) T. Sawada, K. Oikawa, K. Anzai, F. Takahashi, K. Kajikawa and H. Yamada: J. JFS, 81 (2009), 283.

13) Y. Ebisu: Metall. Trans. B, 42B (2011), 341.

14) T. S. Piwonka and M. C. Flemings: Trans. Metall. Soc. AIME, 236 (1966), 1157.

15) D. Apelian, M. C. Flemings and R. Mehrabian: Metall. Trans., 5 (1974), 2533.

16) N. Streat and F. Weinberg: Metall. Trans. B, 7B (1976), 417.

17) K. Murakami and T. Okamoto: Acta Metall., 32 (1984), 1741.

18) K. Murakami, A. Shiraishi and T. Okamoto: Acta Metall., 31 (1983), 1417.

19) K. Murakami, A. Shiraishi and T. Okamoto: Acta Metall., 32 (1984), 1423.

20) C. Y. Liu, K. Murakami and T. Okamoto: Mater. Sci. Technol., 5 (1989), 1148.

21) R. Nasser-Rafi, R. Deshmukh and D. R. Poirier: Metall. Trans., 16A (1985), 2263.

22) D. R. Poirier: Metall. Trans., 18B (1987), 245.

23) D. R. Poirier and P. Ocansey: Mater. Sci. Eng., A171 (1993), 231.

24) S. Ganesan, C. L. Chan and D. R. Poirier: Mater. Sci. Eng., A151 (1992), 92.

25) M. S. Bhat, D. R. Poirier and J. C. Heinrich: Metall. Trans., 26B (1995), 1049.

26) J. C. Heinrich, D. R. Poirier and D. Nagelhout: Comput. Meth. Appl. Mech. Eng., 133 (1996), 79.

27) D. R. Poirier and J. C. Heinrich: Mater. Charact., 32 (1994), 287.

28) J. C. Heinrich and D. R. Poirier: C. R. Mecanique, 332 (2004), 429.

29) D. Fuloria, P. D. Lee and D. Bernard: Mater. Sci. Eng., A494 (2008), 3 .

30) J. Madison, J. Spowart, D. Rowenhorst, L. K. Aagesen, K. Thornton and T. M. Pollock: Acta Mater., 58 (2010), 2864.

31) R. Kobayashi: Physica D, 63 (1993), 410.

32) A. Karma and W.-J. Rappel: Phys. Rev. E, 57(1998), 4323.

33) J. S. Lee and T. Suzuki: ISIJ Int., 39 (1999), 246.

34) Y. Natsume: R\&D Kobe Steel Eng. Rep., 58 (2008), 35.

35) Y. Natsume and H. Ishida: Proc. of 12th ICAA, JILM, Tokyo, (2010), 1746.

36) Kinzoku Databook, Rev. 3, J. Jpn. Inst. Met., Maruzen, Tokyo, (1993), 16

37) T. Takahashi and I. Hagiwara: J. Jpn. Inst. Met., 29 (1965), 1152.

38) T. Takahashi, M. Kudoh and K. Yodoshi: J. Jpn. Inst. Met., 43 (1975), 1086.

39) T. Takahashi, M. Kudoh and S. Nagai: Tetsu-to-Hagané, 68 (1982), 623 . 